\title{
Cloning and functional characterization of the intersex homologous gene in the pest lepidopteron Maruca vitrata
}

\author{
DANIELA CAVALIERE ${ }^{1}$, FRANCESCA DI CARA², LINO C. POLITO ${ }^{1,3}$ and FILOMENA ANNA DIGILIO*,1 \\ ${ }^{1}$ Institute of Genetics and Biophisics "Adriano Buzzati-Traverso"-CNR, Naples, Italy, \\ ${ }^{2}$ Cell Biology, Centre for Cardiovascular Science, The University of Edinburgh-Queen's Medical Research \\ Institute and ${ }^{3}$ Department of Biological Sciences, Section of Genetics and Molecular Biology, University of \\ Naples "Federico II", Naples, Italy
}

\begin{abstract}
The intersex (ix) gene works in concert with doublesex (dsx) at the bottom of the sexdetermination hierarchy to control somatic sexual differentiation in Drosophila melanogaster females. Here we report the isolation and characterization of the Drosophila intersex (ix) homologue in the pest lepidopteron Maruca vitrata (Mvix). The Mvix gene exhibits major complexity with respect to the Drosophila homolog. It is expressed in males and females and its pre-mRNA is subject to differential splicing events which affect both the protein coding and the non-coding regions. Moreover, Northern blot experiments revealed the presence of a femalespecific transcript in pupae RNA, which appears to be the first described sex specific transcript of ix homologs characterized to date. The expression of Mvix cDNA in D.melanogaster transgenic flies indicates that the MvIX product, which shares a relatively high degree of homology with the D.melanogaster IX protein, is able to partially rescues the Drosophila mutant phenotype.
\end{abstract}

KEY WORDS: Maruca vitrata, intersex, sex determination, Drosophila melanogaster

\section{Introduction}

Sex determination cascades that drive the somatic sexual differentiation are some of the most rapidly evolved developmental events (Revised in Fujii and Shimada 2007; Traut et al. 2007). Nevertheless, recent studies indicate that some of the downstream sex determination genes are functionally similar in diverse species. Among these, the doublesex gene is one of the most conserved sex-determining genes and has been reported to regulate sexual development in many metazoan phyla, from worm to human (as revised in Sanchez 2008; Saccone et al. 2008). $d s x$ functions in both sexes; its primary transcript undergoes sex-specific alternative splicing, producing either a malespecific isoform, DSXM, or a female-specific isoform, DSXF, that exhibit similar DNA-binding properties although have opposite biological functions (Baker and Ridge 1980; Coschigano and Wensink 1993).

In Drosophila, DSXF (but not DSXM) coworks with Intersex (IX), and it has been demonstrated that these two proteins interact specifically in vitro and that this binding is required for activation of the yolk protein (Garrett-Engele et al. 2002). In addition to regulating Yp expression, DSXF and IX also cooperate to regulate the female-specific differentiation of sexually dimorphic cuticular structures (Chase and Baker 1995, Garrett-Engele et al. 2002). $d s x$ and ixgenes have both been cloned in different organisms and functionally characterized (Siegal and Baker 2005; Sato et al. 2003). The IX proteins show a conserved organization, with a transcriptional activation domain at the aminoN-terminal region. Heterologous expression of insect and mammalian ix homologs in transgenic Drosophila suggestes that its functional interaction with DSXF is at least in part conserved (Siegal and Baker 2005). Among ixhomologs deriving from insects, the lepidopteron Bombyx mori ix cDNA does not fully restore female differentiation in $D$.melanogaster ix-mutant females. Based on their data, the authors speculate that the B.morilX and D.melanogasterDSXF proteins interact imperfectly and suggest a possibile co-evolution of both products. In order to study this co-evolution within the Lepidoptera order, we planned to isolate these two sex-determining genes in a lepidopteron belonging to a different family with respect to B.mori - that is representative of the Bombycidae family-, and to compare their organization in these two species.

In this paper, we report the identification, cloning and charac-

Abbreviations used in this paper: dsx, doublesex; ix, intersex; MvIX, Maruca vitrata INTERSEX.

\footnotetext{
*Address correspondence to: Dr. Digilio Filomena Anna. Institute of Genetics and Biophisics "Adriano Buzzati-Traverso", Via P. Castellino 111, 80131 Naples, Italy. Fax:+39-081-613-2706. e-mail: digilio@igb.cnr.it
}

Accepted: 7 April 2009. Published online: 19 June 2009. 
A

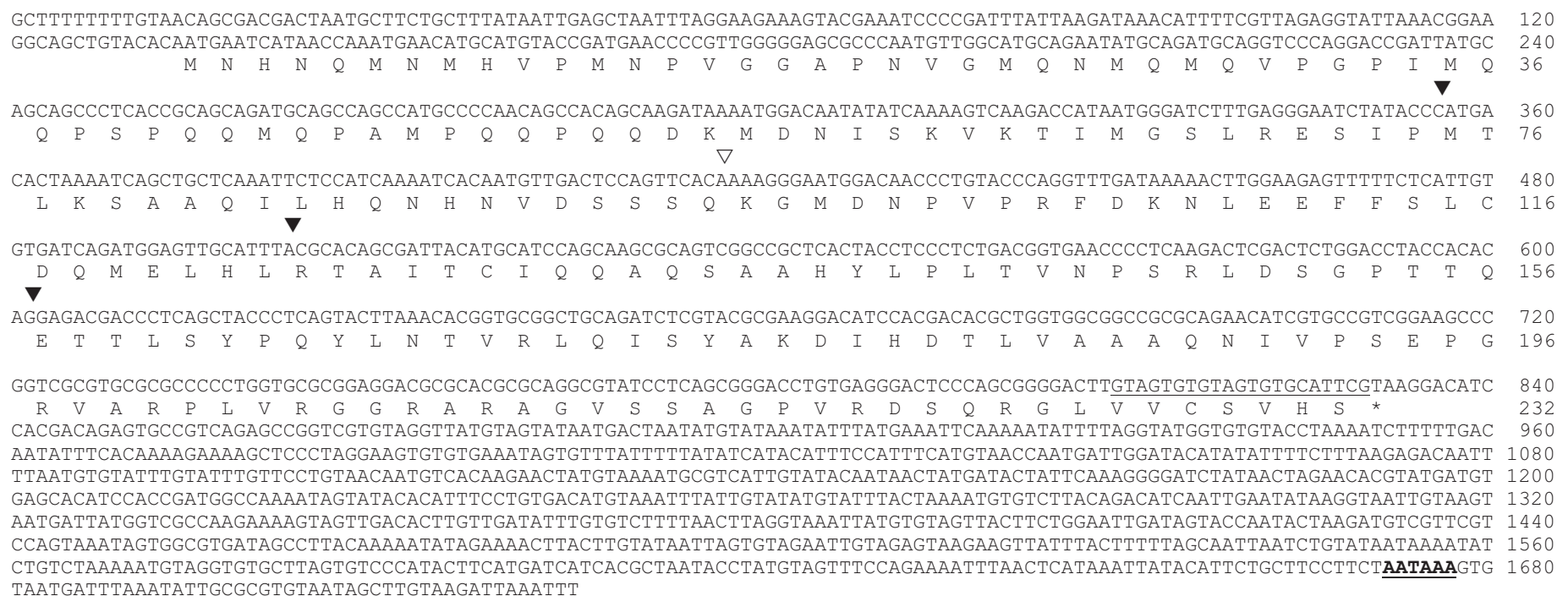

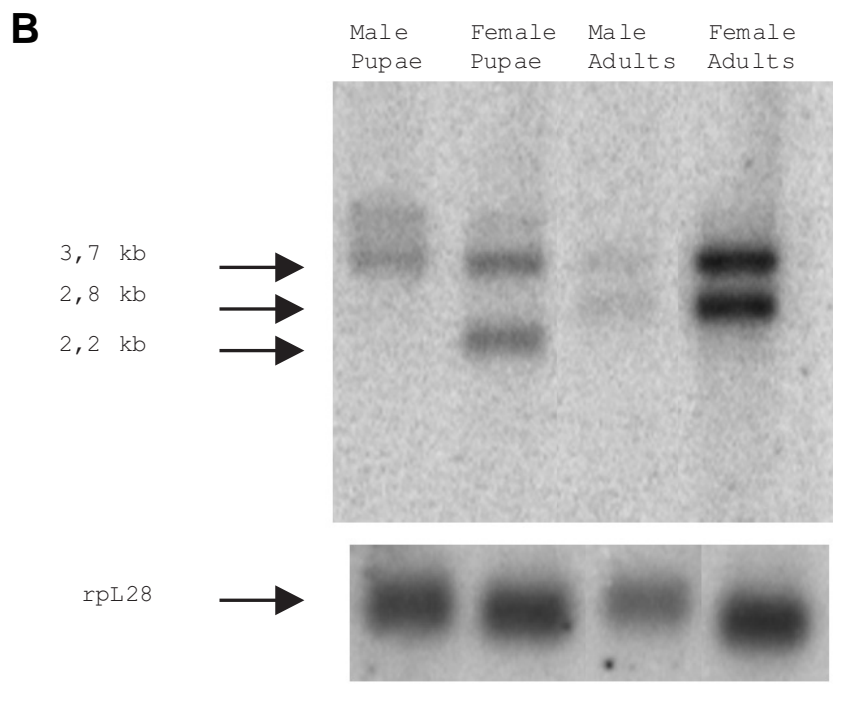

terization of ix homolog in Maruca vitrata, that is a lepidopteron belonging to the Piralidae family. This insect is considered a serious pest of legume crops from tropics to the temperate zone all over the world and is especially recognized as a key pest of cowpea in Africa.

\section{Results and Discussion}

\section{Isolation of the ix homolog in Maruca vitrata}

To identify the ixtranscript in M.vitrata, a cDNA library derived from pupae was screened with the B.mori ix transcript sequence. A total of five clones were isolated and fully sequenced. Their analyses revealed an Open Reading Frame 696 bp long encoding for a 232 aminoacids polypeptide that shows a high degree of similarity with the INTERSEX proteins characterized so far. Among all these homologues, the highest score of similarity was found with the B.morilX protein, with a $86 \%$ identity (Siegal and Baker 2005) (Fig. 1A).

MvIX consists of an aminoN-terminal region of about 47
Fig. 1. Maruca vitrata ix sequence and its expression profile. (A) Mvix DNA sequence (above) and the predicted protein sequence (below). The putative poly $(A){ }^{+}$signal is boldface typed. ${ }^{*}$, termination codon; $\mathbf{\nabla}$, sites of introns; $\nabla$, site of the intron with alternative splicing acceptor site. (B) A Northern blot of poly $(A)^{+} R N A$ extracted from sexed pupae and adults was hybridized with the single-stranded antisense Mvix CDNA. The relative abundance of the transcripts were normalized to the amounts of MvrpL28 transcript (characterized in our laboratory).

residues, that presents the aminoacid composition-rich in glutamine, methionine, proline, glycine and asparagine- which defines the transcriptional activation domain. Downstream to this region, there are several stretches of aminoacid identity that are highly conserved only among the IX homologues. In D.melanogaster, one of these stretches, corresponding to residues 94-116, contains four aminoacids that are important to bind DSXF; this has been recently suggested in mutagenesis experiments in yeast (Yang et al. 2008).

At the carboxyC- terminal region, MvIX protein presents an additional region of 38 aminoacids. This region does not define a known domain and it is just peculiar for MvIX, because it is not conserved among the other IX homologues characterized to date.

\section{Expressional profile and genomic organization of the ix gene in Maruca vitrata}

In D.melanogasterthe ix gene is transcribed in both sexes as a single mRNA that is about 9 times more abundant in wildtype females than in wild-type males. This transcript is not sex-specific and is not spliced (Garrett-Engele et al. 2002). In M. vitrata ix gene presents a major complexity and is transcribed in alternative mRNAs in both sexes. Northern blot analysis, carried out on sexed pupae and adults using the longest Mvix cDNA as ${ }^{32} \mathrm{P}$ labeled probe, revealed different transcripts in these two developmental stages. In both sexes adults the revealed two transcripts, of approximately $3,7 \mathrm{~kb}$ and 2,8 $\mathrm{kb}$, are equimolar, and are $\sim 3$ times more abundant in wild-type females than in wild-type males, as well as in Drosophila (Fig. 1B). In contrast, in pupae of both sexes only the longest transcript was observed. Further, interestingly, another 2,2 kb transcript was present only in female pupae, 
suggesting that Mvixpre-mRNA could be alternatively spliced or transcribed in a sex-specific manner in this developmental stage. To date, there is no counterpart ix homologues transcript with a similar exclusive female-specific expression pattern, and future studies will be needed to determine structure and possible functions of this isoform in female pupae.

All the transcripts identified in the Northern blot experiments were longer than the corresponding length of Mvix cDNA. The longest cDNA clone, $1,8 \mathrm{~kb}$ long, contained a poly $(A)^{+}$tail with a consensus polyadenylation signal in the right position and could be full length at the 3' end where is present an untranslated region of $959 \mathrm{bp}$ (Fig. 1A). In order to determine the 5' end, we utilized 5'RACE assays, but unfortunately we were unable to extend the cDNA clone more than $40 \mathrm{bp}$.

However, our Northern blot analyses with probes corresponding to different regions of our cDNA clone, still suggest that all the three transcripts share the coding region (data not shown). To confirm that the transcripts have identical coding regions, RTPCR analysis was performed on poly $(A)^{+}$RNA extracted from sexed pupae and adults, using a pair of specific primers encompassing the ORF. We detected the same product in both sexed pupae and adults, indicating that the Mvix gene encodes always the same protein. However, sequence analysis of the amplified products indicated the presence of two transcripts in adult males, that are produced from a Mvix pre-mRNA using two different acceptor sites in the second intron (Fig. 2 A,B). These transcripts differ at level of the third exon, where the alternative mRNA contains an additional $17 \mathrm{bp}$ long region, that introduces a shift in the MvIX ORF. The hypotethical encoded protein is 148 aa long and shares the first 94 aa with MvIX protein; the C-terminal region, 53 aa long, does not define any known domain and does not produce significant similarity in databases. RT-PCR reactions, conducted with two different pairs of primers specific for each of the two alternative splicing sites, indicated that both transcripts are present in sexed pupae and adults, and that the transcript coding for the shorter form is present at very low levels with respect to the $i x$ coding transcript (Fig. 2C).

As revealed by a Southern blotting experiment, Mvix gene is present in single copy per haploid genome (data not shown). The amplification of the genomic region corresponding to the coding sequence using a pair of primers encompassing the ends of the ORF, and its comparison with the transcripts revealed that the coding region of Mvix gene expands in a chromosomal region of $8 \mathrm{~kb}$ with 5 exons-4 introns (Fig. 1A).

\section{Comparison with the Bombyx mori ix gene}

The Mvix gene presents a major genic complexity with respect to the Drosophila homolog, since no introns were identified. In order to define whether this complexity could be conserved in Lepidoptera, we analyzed the organization of ix gene in B. mori, based on the high degree of homology shared between Bombyx and Maruca ixgene and taking advantage of the Bombyxgenomic sequence available in the data bases (Xia et al. 2004; Wang et al. 2005).

By analyzing the ESTs BB982766, BP180119, BP127552, that correspond to the $i x$ gene in the $B$. mori EST database (http://

A

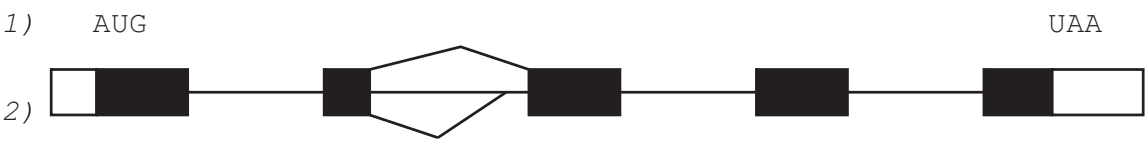

B

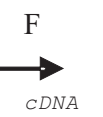

ACACTAAAATCAGCTGCTCAAATTCTCCATCAAAATCACAATGTTGACTCCAGTTCACAgtga-//-tttagtattttctttattcCagAAAGGGAATGGACAACCCTGTACCC

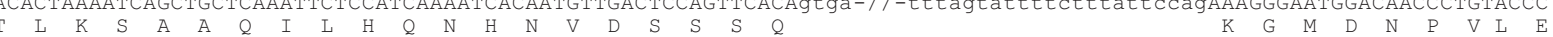

\begin{tabular}{|c|c|c|c|}
\hline \multicolumn{2}{|c|}{ MALE } & \multicolumn{2}{|c|}{ FEMALE } \\
\hline $\mathrm{F}+\mathrm{R} 1$ & $\mathrm{~F}+\mathrm{R} 2$ & $\mathrm{~F}+\mathrm{R} 1$ & $\mathrm{~F}+\mathrm{R} 2$ \\
\hline
\end{tabular}

Fig. 2. The Mvix alternatively spliced mRNA variants. Scheme (A) and nucleotide sequence (B) of the two transcripts (1 and 2) alternatively spliced from Mvix pre-mRNA at two different acceptor sites in the second intron (lower-case). Arrows indicate the primers $F$, R1 and R2 designed according to the nucleotide sequence of each transcript. (C) RT-PCR products obtained with sexed adults and pupae (not shown) mRNA, using different pairs of primers shown in (B). 
www.ab.a.u-tokyo.ac.jp/silkbase/), we defined two different, partially overlapping, transcripts in ovaries and testes. Compared to the ovary transcript, the testis clone contains two additional regions, $160 \mathrm{bp}$ and $89 \mathrm{bp}$ long, at the 5' and the CDS regions level, respectively (Fig. 3A). To determine the exon/intron organization of the Bmix gene, we blasted the $B$. mori genomic database with these two assembled ESTs, obtaining an unique genomic clone, $\mathrm{CH} 386538.1$, localized on chromosome 7, that showed a perfect match with the CDS region of both transcripts. The testis specific 5' UTR defines a different genomic scaffold CH394514.1, which suggests a transcription start point, for this transcript, far away on the genomic region.

The obtained results indicate that the ix CDS region in Bombyx spans at least $8 \mathrm{~kb}$ and that the exon-intron organization is conserved between Maruca and Bombyx with 5 exons and 4 introns. Further, the comparison of the two transcripts sequences to the ix DNA sequence, revealed that the region of $89 \mathrm{bp}$, that is only present in the testis specific EST, BP127552, defines an extra exon at level of the second intron. To test the possibility that this transcript could be sex specific, we carried out a RT-PCR reaction, with $\mathrm{mRNAs}$ extracted separately from head, thorax and abdomen of sexed adults. This analysis identified two mRNAs that are produced, by alternative splicing, from a Bmix pre-mRNA (Fig. 3 $A, B)$. Although it seems that the transcript with the extra exon is more abundant in abdomen of adult males, there is no sex specificity in this alternative splicing. The extra exon introduces a stop codon in the $i x \mathrm{CDS}$, leading to a truncated protein, that shares all its 72 aa with BmIX protein. (Fig. 3B).

In summary, while we have no idea whether also in B.morias in M. vitrata is present a sex specific transcript in pupae, our data suggest that, differently from Drosophila, in these two Lepidoptera, ixpre-mRNA is subject to alternative splice events that affect both the protein coding and the non-coding regions. These alternative splicing events could originate a truncated form of the IX protein and/or could result in changes in regulatory elements such as translation enhancers or RNA stability domains, suggesting a final effect on the level of protein expression. To date, there is still little molecular information on the ix transcripts, so further investigations at the molecular level should lead to finer understanding of the role of the IX product in the regulation of the sex determination process.

\section{Functional conservation by transgenic test}

To test for functional conservation of the Mvix coding sequence,

A

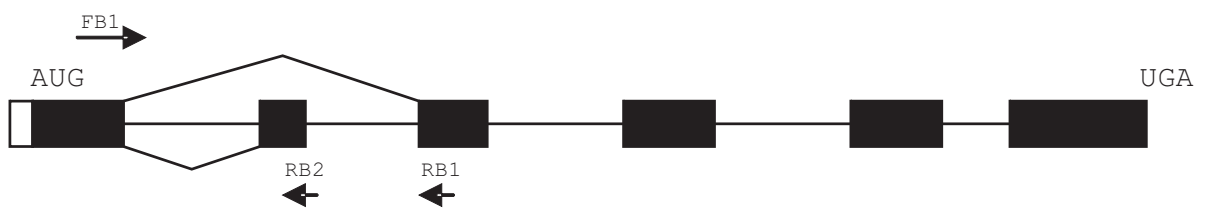

B

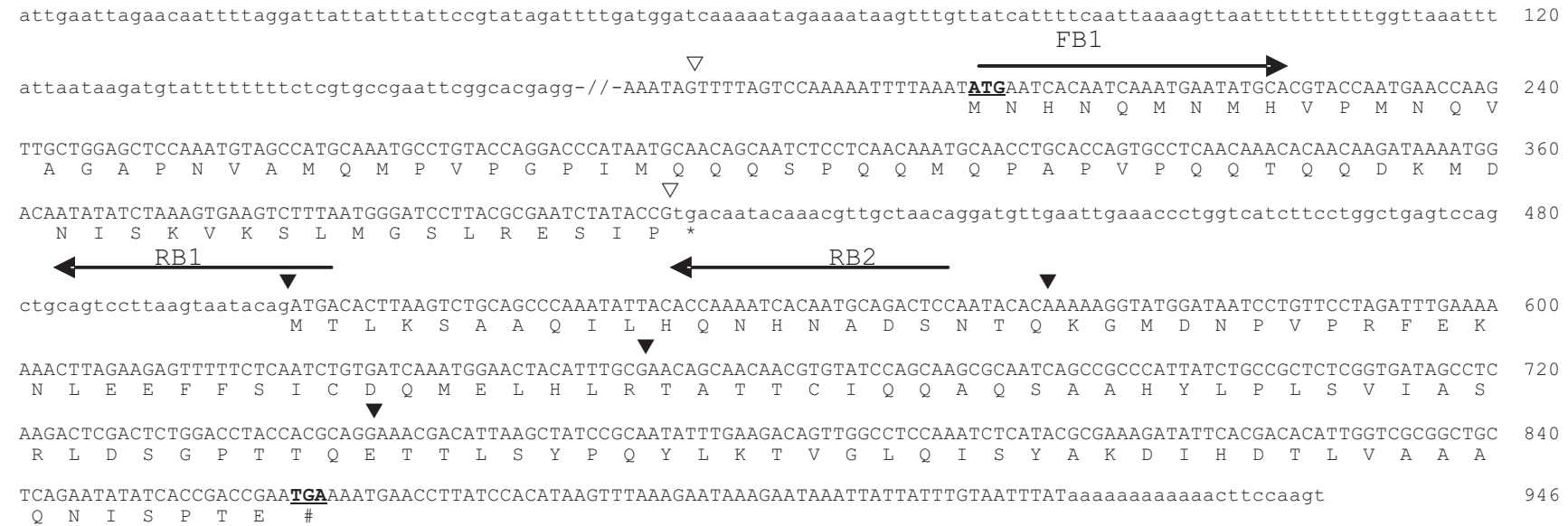

C

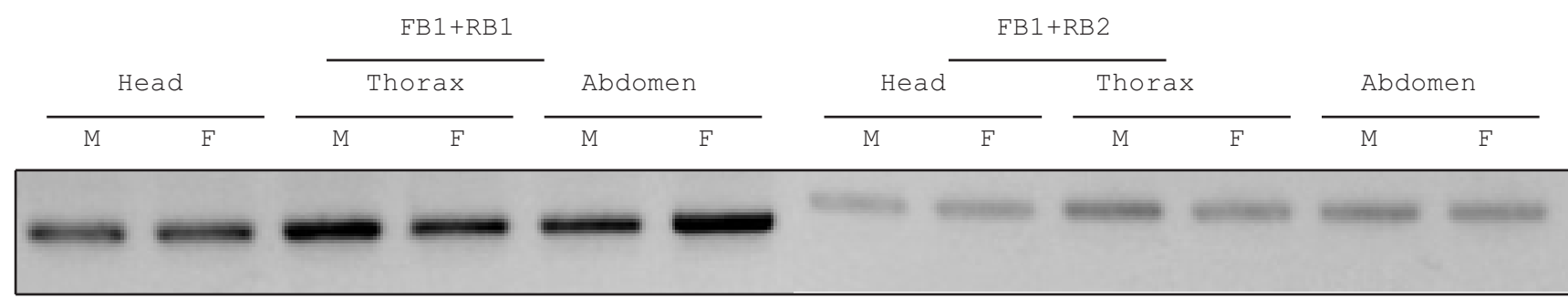

Fig. 3. B.mori ix DNA and its predicted protein sequence. Scheme (A) and nucleotide sequence (B) ot the Bmix DNA with its predicted protein sequence (below). $\mathbf{\nabla}$, site of intron; $\nabla$, site of intron with alternative splicing acceptor site, ${ }^{*}$ stop codon of the alternative polypeptide. The testis specific EST BP127552 contains two additional regions (lowercase). Arrows indicate the primers FB1, RB1 and RB2 designed according to the nucleotide sequence of each transcript. (C) RT-PCR products found in sexed head, thorax and abdomen mRNA, using different primer pairs shown in (A). 

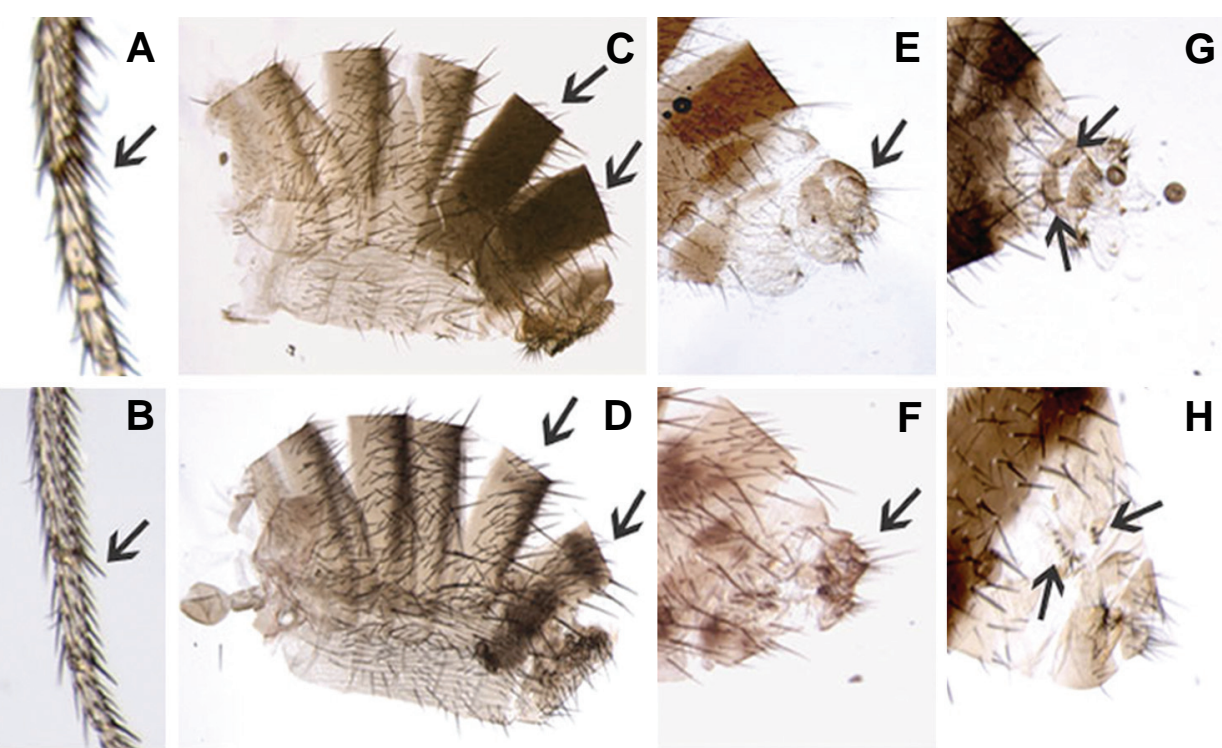

Fig. 4. Morphological characters of D.melanogasterfemales expressing Mvix cDNA. The upper line shows the cuticular structures of $\mathrm{ix}^{1} / 1 \mathrm{X}^{2}$ mutant females. The lower line shows the cuticular structures of $\mathrm{ix} 1 / \mathrm{ix}^{2}$ mutant females expressing Mvix cDNA. Arrows indicate the LTRB in (A,B), the

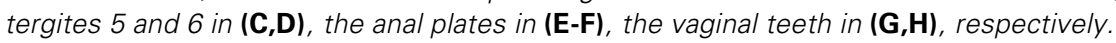

the other transverse rows, but with no rotation (Fig. 4B).

Drosophilamales show a typical dark pigmentation on the abdominal tergites 5 and 6 that is lacking in females, while ix mutant females manifest a male-like pigmentation (Fig. 4C). When these mutant females express Mvix, they revert to a female-like pigmentation in tergites 5 and 6 (Fig. 4D).

In wildtype Drosophila, the anal plates develop differently in both sexes. Females have one dorsal and one ventral anal plate, while males have one left and one right anal plate. ix mutant females do not completely develop the ventral plate and present the dorsal plate splitted and shifted laterally, which results in a similar structure to males (Fig. 4E). Mvix only partially rescues this phenotype, leading to mostly female-like anal plates (Fig. 4F).

In Drosophila the genital bristles, known as vaginal teeth, are found only in wildtype females as two parallel rows on

we used the GAL4-UAS binary system (Brand and Perrimon 1993) to drive the expression of MvixcDNA in transgenic D.melanogaster females mutant for the endogenous ix gene. To compare our results with those obtained with $B$. mori ix by Siegal and Baker, we decided to utilize similar experimental conditions (Siegal and Baker 2005). D. melanogaster and $M$. vitrata ix coding regions were cloned downstream of the UAS in pUASp (Rorth 1998), and activated by GAL4 under tubulin 1apromoter ( $\alpha$ Tub84B), which is constitutively expressed throughout development. The experiments were carried out testing three independent UASp insertions and identical results were observed in all the lines.

The genetic background we used in our experiment was a transheterozygotic combination of two hypomorph alleles $\left(i x^{1}\right)$ $\left.i x^{2}\right)$. The mutant females are sterile and display a strong mutant phenotype at the level of the sexually dimorphic cuticular structures examined by Siegal and Baker. The expression of $D$. melanogaster ix cDNA in these mutant females led to a complete rescue of this phenotype and fertility in our control transgenic lines (data not shown). On the other hand, expressing the Mvix cDNA still resulted in sterile females and the characteristic morphological defects were rescued with different variability. In our experiments we were not able to test the effect of two copies of the UAS recombinant construct, because higher dosage of MvIX protein seems to reduce the viability of both sexes in the tested transgenic lines.

The first phenotype we have analyzed concerns the Last Transversal Row of Bristles on the basitarsus of the foreleg (LTRB). In wildtype males it arranges to form the sex comb, while in wildtype female it is indistinguishable from the other bristle rows on the leg segment. As shown in Fig. 4A, i $x^{1} / i x^{2}$ mutant females develop an intermediate phenotype, whith the LTRB that is thicker, darker and partially rotated than the corresponding female bristles. The expression of Mvix in these females lead to a partially rescued LTRB that is only slightly darker and thicker than both sides of the vaginal opening. ix mutant females present a dramatic disorganization and reduction in the number of these bristles (Fig. 4G). This defect is only partially recovered by expressing Mvix, since the formation of two rows of vaginal teeth show several gaps (Fig. 4H).

In summary, as shown by Siegal et al. on Bmix (2005), also MvIX protein is able to partially replace the Drosophila endogenous IX protein. Significantly, the expression of MvixcDNA in $D$. melanogaster transgenic flies resulted in various degrees of rescue on the examined characters, but never rescued Drosophila fertility. These data might reflect a reduced affinity of the $B m I X$ and MuX proteins for Drosophila DSXF, since only two of the four aminoacids that impair the binding with DSXF in $D$. melanogaster (Yang et al. 2008) are conserved.

In summary, we have cloned and characterized Mvix cDNA. This work suggests that the regulatory mechanism of ixin M. vitrata and B.moriappears to be very complicated, and it also represents the first molecular report of a gene of M.vitrata that will certainly help to understand basic aspects of sex-determining genes in a lepidopteron causing serious damage to the cowpea crops.

\section{Materials \& Methods}

\section{Fly strains}

Stocks were provided by the Bloomington Stock Center, and were raised at $25^{\circ} \mathrm{C}$ under standard conditions.

\section{Isolation and sequencing of cDNA clones}

Mvix cDNAs were isolated by screening a pupae cDNA $\lambda$ ZAP library prepared in our laboratory, with the $B$. mori ix $\mathrm{CDS}$ as ${ }^{32} \mathrm{P}$-labelled probe. Both strands of cDNA inserts were sequenced at Primm Biotech- Naples.

\section{Southern analysis}

Genomic DNA was isolated, electrophoresed, transferred and probed using standard techniques (Sambrook et al., 1989). 


\section{Northern analysis}

Total RNA was extracted using TRI reagent (Sigma), and poly(A)+ RNA samples were prepared using Qiagen mRNA Purification Kit following the manufacturer's instructions. Northern blot was carried out according to Di Cara et al. 2006. The ${ }^{32} \mathrm{P}$ labeled ixprobe was prepared by singlestranded PCR with primer Rev CDS (underlined nucleotides 810-830 in Fig. 1A).

\section{Cloning of UAS-Mvix, P-element-mediated germline transformation and cuticle preparations}

Mvix was cloned downstream of the UAS promoter into the vector pUASp (Rorth 1998). Transgenic Drosophila flies were generated by standard P-element mediated germline transformation of Drosophila embryos (Rubin and Spradling 1982). Cuticle preparations were obtained according to Siegal and Baker 2005.

\section{$R T-P C R$ and 5'RACE}

$1 \mu \mathrm{g}$ of poly $(\mathrm{A})+$ mRNA was reverse transcripted by Stratascript reverse transcriptase III (Stratagene) and a poly-dT primer. PCR and 5 RACE reactions were performed using the EuroTaq (Euroclone), and the Marathon cDNA Amplification Kit (Clontech Laboratories, Inc.), and the products were cloned into pGemT-easy vector (Promega).

\section{Acknowledgements}

This work was supported by a grant from the Regione Campania (L.R. 31.12.1994 n.5) to C.L.P. We are greatiful to Manu Tamo for kindly sending us M.vitrata sexed pupae. We thank Angela Sorrentino for having isolated and partially characterized the CDNA clone. In addition, we like to thank Annamaria De Simone for her help with 5'RACE experiments.

\section{References}

BAKER, B.S. and K.A. RIDGE. (1980). Sex and the single cell. I. On the action of major loci affecting sex determination in Drosophila melanogaster. Genetics 94 : 383-423.

BRAND, A.H. and N. PERRIMON. (1993). Targeted gene expression as a means of altering cell fates and generating dominant phenotypes. Development 118 : 401-415.

CHASE, B.A. and B.S. BAKER. (1995). A genetic analysis of intersex, a gene regulating sexual differentiation in Drosophila melanogasterfemales. Genetics 139: $1649-1661$.

COSCHIGANO, K.T. and P.C. WENSINK. (1993). Sex-specific transcriptional regulation by the male and female doublesex proteins of Drosophila. Genes \& Development 7: 42-54.

DI CARA, F., R. MORRA, D. CAVALIERE, A. SORRENTINO, A. DE SIMONE, C.L. POLITO, and A.F. DIGILIO. (2006). Structure and expression of a novel gene family showing male germline specific expression in Drosophila melanogaster. Insect molecular biology 15: 813-822.

FUJII, T. and T. SHIMADA. (2007). Sex determination in the silkworm, Bombyx mori: a female determinant on the $\mathrm{W}$ chromosome and the sex-determining gene cascade. Sem. Cell Dev. Biol.18: 379-388.

GARRETT-ENGELE, C.M., M.L. SIEGAL, D.S. MANOLI, B.C. WILLIAMS, H. LI, and B.S. BAKER. (2002). intersex, a gene required for female sexual development in Drosophila, is expressed in both sexes and functions together with doublesex to regulate terminal differentiation. Development 129: 4661-4675.

RORTH, P. (1998). Gal4 in the Drosophila female germline. Mech. Dev. 78:113118.

RUBIN, G.M. and A.C. SPRADLING. (1982). Genetic transformation of Drosophila with transposable element vectors. Science 218: 348-353.

SACCONE, G., M. SALVEMINI, A. PANE, and L.C. POLITO. (2008). Masculinization of XX Drosophila transgenic flies expressing the Ceratitis capitata DoublesexM isoform. Int. J. Dev. Biol. 52: 1051-1057.

SAMBROOK, J., E.F. FRITSCH, and T. MANIATIS. (1989). Molecular cloning: a laboratory manual. Cold Spring Harbor, NY: Cold Spring Harbor Laboratory Press.

SANCHEZ, L. (2008). Sex-determining mechanisms in insects. Int. J. Dev. Biol. 52: 837-856.

SATO, S., C. TOMOMORI-SATO, C.A. BANKS, T.J. PARMELY, I. SOROKINA, C.S. BROWER, R.C. CONAWAY, and J.W. CONAWAY. (2003). A mammalian homolog of Drosophila melanogaster transcriptional coactivator intersex is a subunit of the mammalian Mediator complex. J. Biol. Chem. 278: 49671-49674

SIEGAL, M.L. and B.S. BAKER. (2005). Functional conservation and divergence of intersex, a gene required for female differentiation in Drosophila melanogaster. Dev. Genes Evol. 215: 1-12.

TRAUT, W., K. SAHARA, and F. MAREC. (2007). Sex chromosomes and sex determination in Lepidoptera. Sex Dev1: 332-346

WANG, J., Q. XIA, X. HE, M. DAI, J. RUAN, J. CHEN, G. YU, H. YUAN, ET AL. (2005). SilkDB: a knowledgebase for silkworm biology and genomics. NuC. Acids Res. 33: D399-402.

XIA, Q., Z. ZHOU, C. LU, D. CHENG, F. DAI, B. LI, P. ZHAO, X. ZHA, ET AL. (2004) $A$ draft sequence for the genome of the domesticated silkworm (Bombyx mori). Science 306: 1937-1940.

YANG, Y., W. ZHANG, J.R. BAYRER, and M.A. WEISS. (2008). Doublesex and the regulation of sexual dimorphism in Drosophila melanogaster. structure, function, and mutagenesis of a female-specific domain. J. Biol. Chem. 283:72807292 . 


\section{Further Related Reading, published previously in the Int. J. Dev. Biol.}

See our recent Special Issue Epigenetics \& Development edited by Saadi Khochbin and Stefan Nonchev at: http://www.ijdb.ehu.es/web/contents.php?vol=53\&issue=2-3

Ceratitis capitata transformer-2 gene is required to establish and maintain the autoregulation of Cctra, the master gene for female sex determination

Marco Salvemini, Mark Robertson, Benjamin Aronson, Peter Atkinson, Lino C. Polito and Giuseppe Saccone Int. J. Dev. Biol. (2009) 53: 109-120

Masculinization of XX Drosophila transgenic flies expressing the Ceratitis capitata DoublesexM isoform

Giuseppe Saccone, Marco Salvemini, Attilio Pane and Lino C. Polito Int. J. Dev. Biol. (2008) 52: 1051-1057

Sex-determining mechanisms in insects

Lucas Sánchez

Int. J. Dev. Biol. (2008) 52: 837-856

Developmental biology for undergraduate students at the University of Palermo, Italy

Giovanni Giudice and Karoly Onorato

Int. J. Dev. Biol. (2003) 47: 123-133

Musca domestica, a window on the evolution of sex-determining mechanisms in insects

Andreas Dübendorfer, Monika Hediger, Géza Burghardt and Daniel Bopp Int. J. Dev. Biol. (2002) 46: 75-79

5 yr ISI Impact Factor $(2008)=3.271$

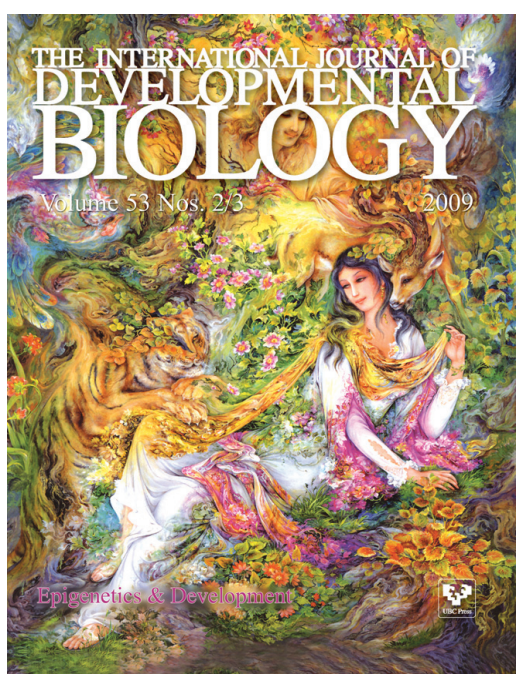

